\title{
Grobdimensionierung des Lastaufnahmerahmens des Trans- portluftschiffes CL 160 in Metall- und Faserverbundbauweise mittels Finite-Elemente-Analyse
}

\author{
Lars Davideit, Marco Schwarz, Peter Selke
}

\section{Einleitung}

Die Finite-Elemente-Methode (FEM) - zutreffender Finite-Elemente-Analyse - ist heute fuir jeden Konstrukteur und Berechnungsingenieur zu einem unverzichtbaren Standardwerkzeug geworden, mit dem man - neben der historischen „Grundaufgabe“ der Analyse von statischen Tragwerken - Feldprobleme für die unterschiedlichsten Anwendungen lösen kann.

Aus diesem Grunde ist dieses von Ingenieuren für Ingenieure entwickelte Verfahren ein Bestandteil des Lehrangebotes für Studenten der Fachrichtung Maschinenbau an der Technischen Fachhochschule Wildau seit Beginn der Ausbildung an dieser Einrichtung vor zehn Jahren (siehe dazu auch [1]).

In dieser Zeit wurden die unterschiedlichsten Probleme, meist aus dem Gebiet der Elastizitätstheorie/Festigkeitsberechnung, aber auch Probleme wie die Untersuchung des Ausbreitungsverhaltens und der Grundwasserbeeinträchtigung von Weichgelsohlen, welches zur Trockenhaltung von Baugruben in den Untergrund eingebracht wird, untersucht [2].
Die Ergebnisse der hier vorgestellten Untersuchung, die eine erste Grobabschätzung der Strukturmasse des Laderahmens für ein in dieser Form neues Transportsystem zum Inhalt hat, entstanden im Rahmen von Diplomarbeiten [3], [4] für die Cargo-Lifter Development GmbH in Briesen-Brand.

\section{Problembeschreibung}

Schwerlasttransporte von der Straße zu lösen ist der Wunsch vieler Unternehmen, da der Logistikaufwand bei extrem großvolumigen oder besonders schweren Transportgütern sehr hoch ist. Oft müssen Straßen erst gebaut, Brücken verstärkt oder beim Transport störende Ampeln und Stromleitungen demontiert werden.

Mit der Entwicklung des Transportluftschiffes CL 160 der Firma Cargo-Lifter AG (Abb. 1) wird das Ziel verfolgt, derartige Güter unkomplizierter und kostengünstiger auch in Gegenden mit geringer Bodeninfrastruktur punktgenau abzusetzen (siehe hierzu auch [í]).

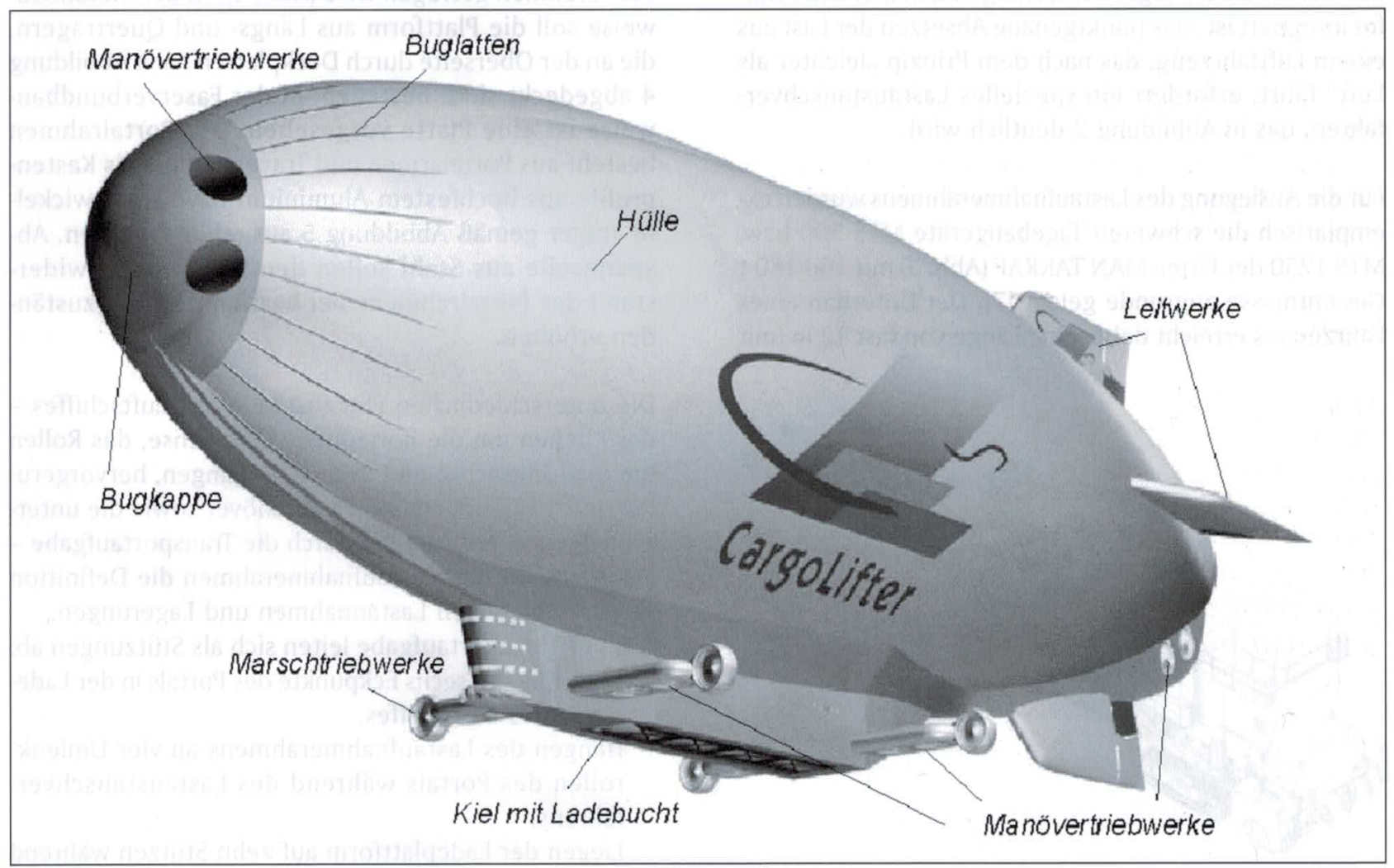




\section{- Beschreibung des Lastaustauschverfahrens}

- Luftschiffpositionierung in ca. $100 \mathrm{~m}$ Höhe über Absetzstelle

- Entriegelung des Laderahmens aus Verankerung der Ladebucht

- Absenkung des Laderahmens auf ca. $40 \mathrm{~m}$

- Aufnahme der Ankerseile durch Hilfswinden

- punktgenaues Absetzen des Laderahmens

- Austausch der Ladung bzw. des Ballastwassers
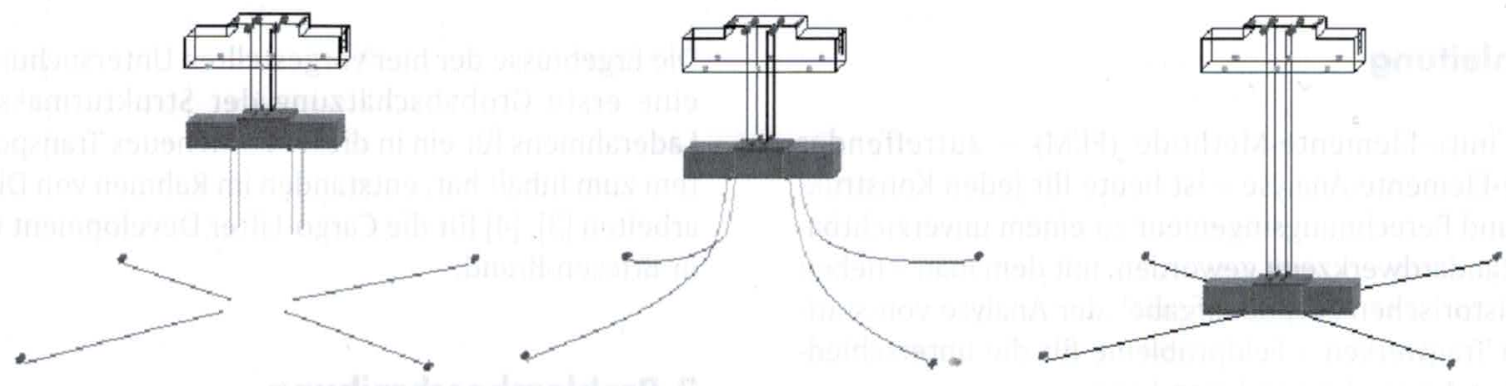

Abb. 2: Lastaustauschverfahren

Bei dem Cargo-Lifter CL 160 handelt es sich um ein halbstarres Luftschiff, $260 \mathrm{~m}$ lang, $65 \mathrm{~m}$ Durchmesser, befüllt mit $550.000 \mathrm{~m}^{3}$ nicht brennbarem Helium, das in der Lage sein soll, $160 \mathrm{t}$ Nutzlast bei einer Reisegeschwindigkeit von $80 \ldots 120 \mathrm{~km} / \mathrm{h}$ in einer Flughöhe von maximal $2.000 \mathrm{~km}$ nonstop über mehrere tausend Kilometer zu befördern. Die Marschgeschwindigkeit soll durch vier Gasturbinen von je $1.700 \mathrm{~kW}$ Leistung, die Propeller von 6,5 m Durchmesser antreiben, erreicht werden.

Im mittleren Teil des Kiels in der Ladebucht (siehe Abb. 1) befindet sich ein Lastaufnahmerahmen $50 \mathrm{~m} \times 8 \mathrm{~m} \times 8 \mathrm{~m}$, der während des Fluges vollständig in den Kiel des Schiffes integriert ist. Das punktgenaue Absetzen der Last aus einem Luftfahrzeug, das nach dem Prinzip „leichter als Luft" fährt, erfordert ein spezielles Lastaustauschverfahren, das in Abbildung 2 deutlich wird.

Für die Auslegung des Lastaufnahmerahmens wurden exemplarisch die schweren Tagebaugeräte MTS 500 bzw. MTS 1250 der Firma MAN TAKRAF (Abb. 3) mit 160/180 t Gesamtmasse zugrunde gelegt [7]. Der Unterbau eines Fahrzeuges erreicht dabei eine Länge von fast $12 \mathrm{~m}$ (mit

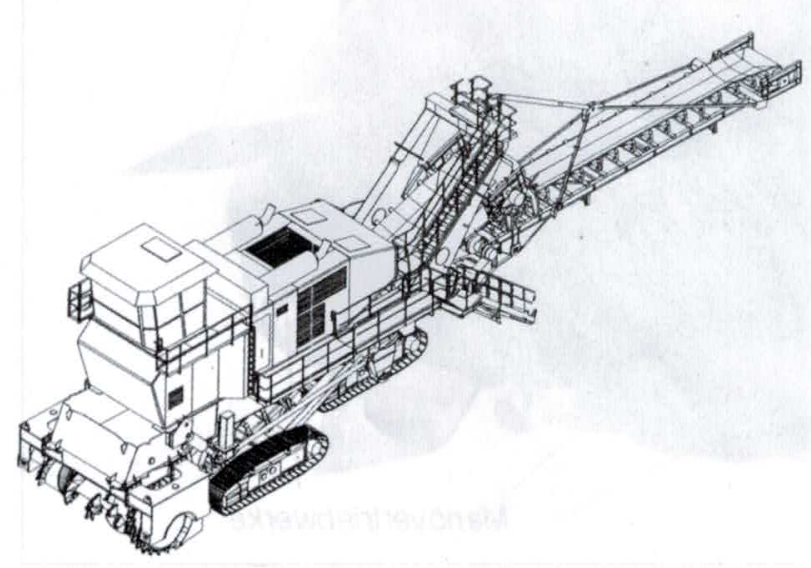

Abb. 3: Tagebaugerät MTS 500 [7]
Förderband und Schneidewerkzeug ca. $30 \mathrm{~m}$ ), die Breite beträgt 4,5 m. Die Höhe der Einheit kann durch das Absenken der Führerkabine von knapp $10 \mathrm{~m}$ auf ca. $7 \mathrm{~m}$ reduziert werden.

\section{Auslegung der Strukłurelemente}

Der Lastaufnahmerahmen des Luftschiffes soll Transportguiter bis zu einer Länge von $50 \mathrm{~m}$ bei einer Breite und Höhe von bis zu 8 m aufnehmen können. Das Konzept sieht dazu eine Ladeplattform vor, die von einem Portalrahmen getragen wird (Abb. 4). In der Metallbauweise soll die Plattform aus Längs- und Querträgern, die an der Oberseite durch Deckplatten nach Abbildung 4 abgedeckt sind, bestehen; in der Faserverbundbauweise ist eine Platte vorgesehen. Der Portalrahmen besteht aus Portalarmen und Traversen, die als Kastenprofile aus hochfestem Aluminium bzw. als gewickelte Träger gemäß Abbildung 5 ausgefuihrt werden. Abspannseile aus Stahl sollen den Verwindungswiderstand des Portalrahmens bei bestimmten Flugzuständen erhöhen.

Die unterschiedlichen Flugzustände des Luftschiffes das Pitchen um die horizontale Querachse, das Rollen um die Längsachse und Beschleunigungen, hervorgerufen durch Windböen und Flugmanöver sowie die unterschiedlichen Belastungen durch die Transportaufgabe erfordern für den Lastaufnahmerahmen die Definition von verschiedenen Lastannahmen und Lagerungen.

Aus der Transportaufgabe leiten sich als Stuitzungen ab:

- Lagerung der sechs Eckpunkte des Portals in der Ladebucht des Luftschiffes,

- Hängen des Lastaufnahmerahmens an vier Umlenkrollen des Portals während des Lastaustauschverfahrens,

- Liegen der Ladeplattform auf zehn Stützen während des Lastaustauschverfahrens. 


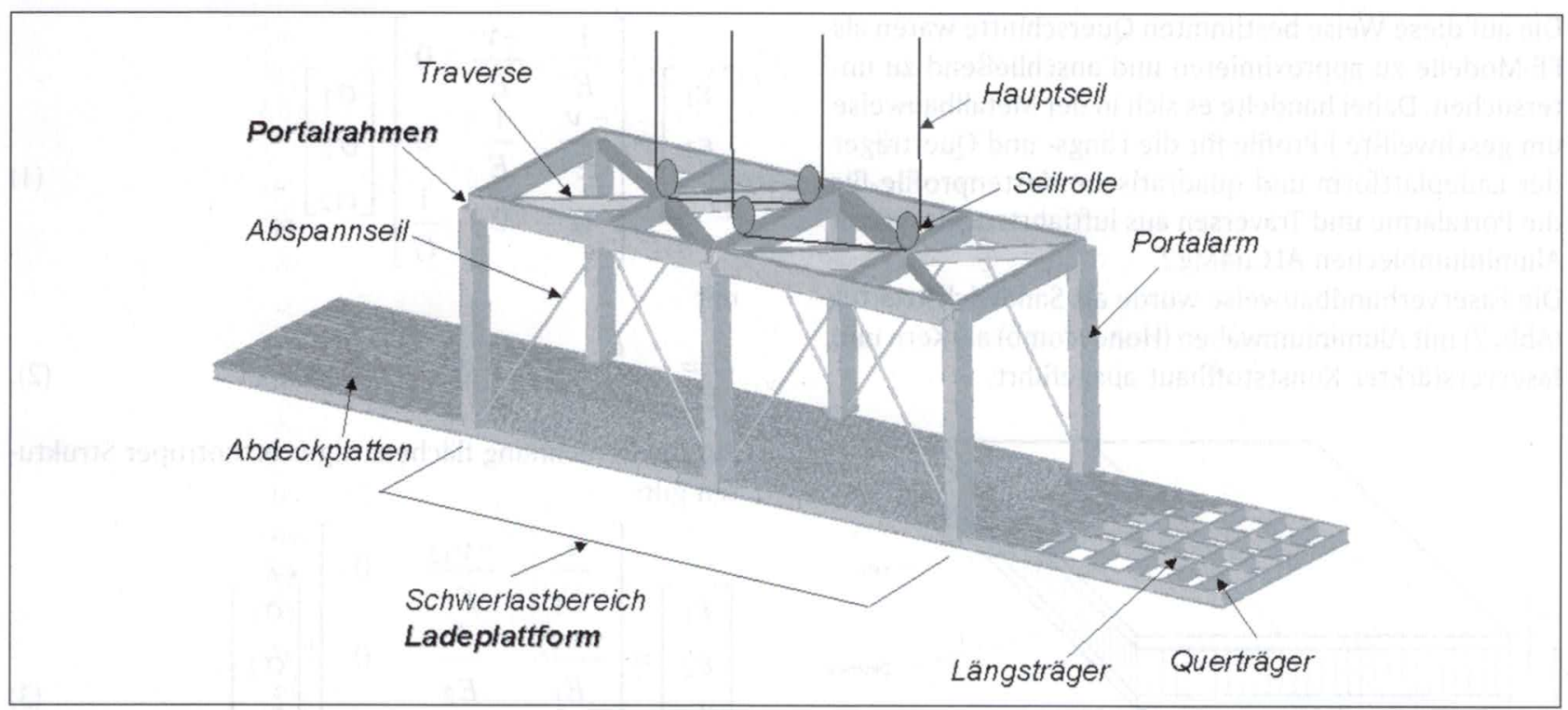

Abb. 4: FEM-Modell des Lastaufnahmerahmens in Metallbauweise [4]

Die Lastfälle:

- Einholen/Herablassen des Ladesystems

- freies Hängen des Lastaufnahmerahmens bei Berüicksichtigung von Beschleunigungen durch Böen, bei Auftrieb durch Manövriertriebwerke und bei thermischem Auftrieb

- Pitchen des Luftschiffes um $25^{\circ}$ bei $1,5 \mathrm{~g}$ Vertikalbeschleunigung nach oben

- Rollen des Luftschiffes um $10^{\circ}$ bei 1,5 g Vertikalbeschleunigung nach oben

sowie einige Lastfallkombinationen ergeben sich aus dem Lastaustauschverfahren und den unterschiedlichen Flugzuständen.

Das prinzipielle Vorgehen bei der Grobauslegung der Strukturelemente wird aus Abbildung 6 ersichtlich. Die Grobauslegung „von Hand“ erfolgte dabei iterativ anhand vorgegebener Verformungs- oder allgemeiner Leichtbaukriterien $[9|\ldots|$ 12]. Für die Faserverbund-Variante erfolgten diese Berechnungen nach - für Kunststoffe typischen - Versagenskriterien [13] bei Beachtung von Verträglichkeiten zwischen den verschiedenen Werkstoffen und Klebern unter Verwendung geeigneter Berechnungssoftware [14].

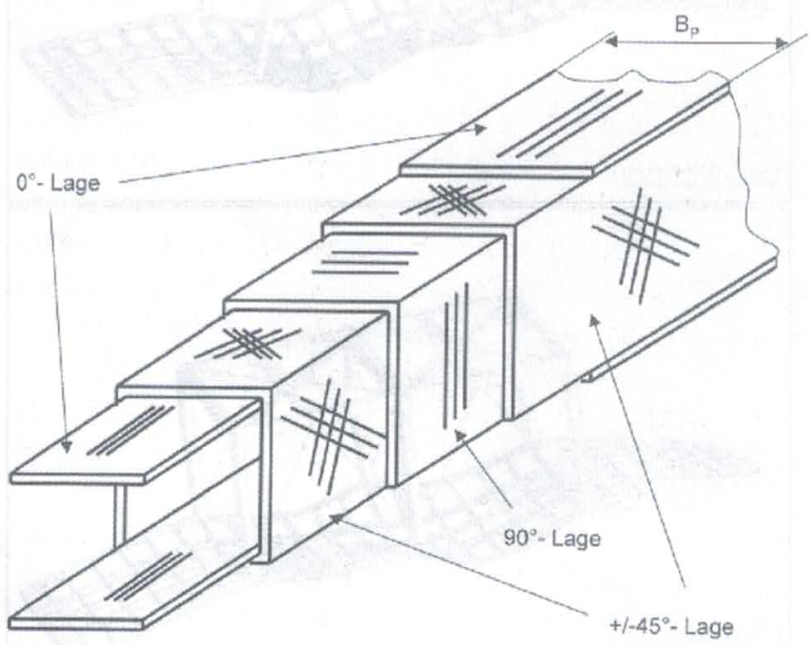

Abb. 5: Typischer Aufbau eines gewickelten Trägers aus Faserverbundwerkstoff [8]

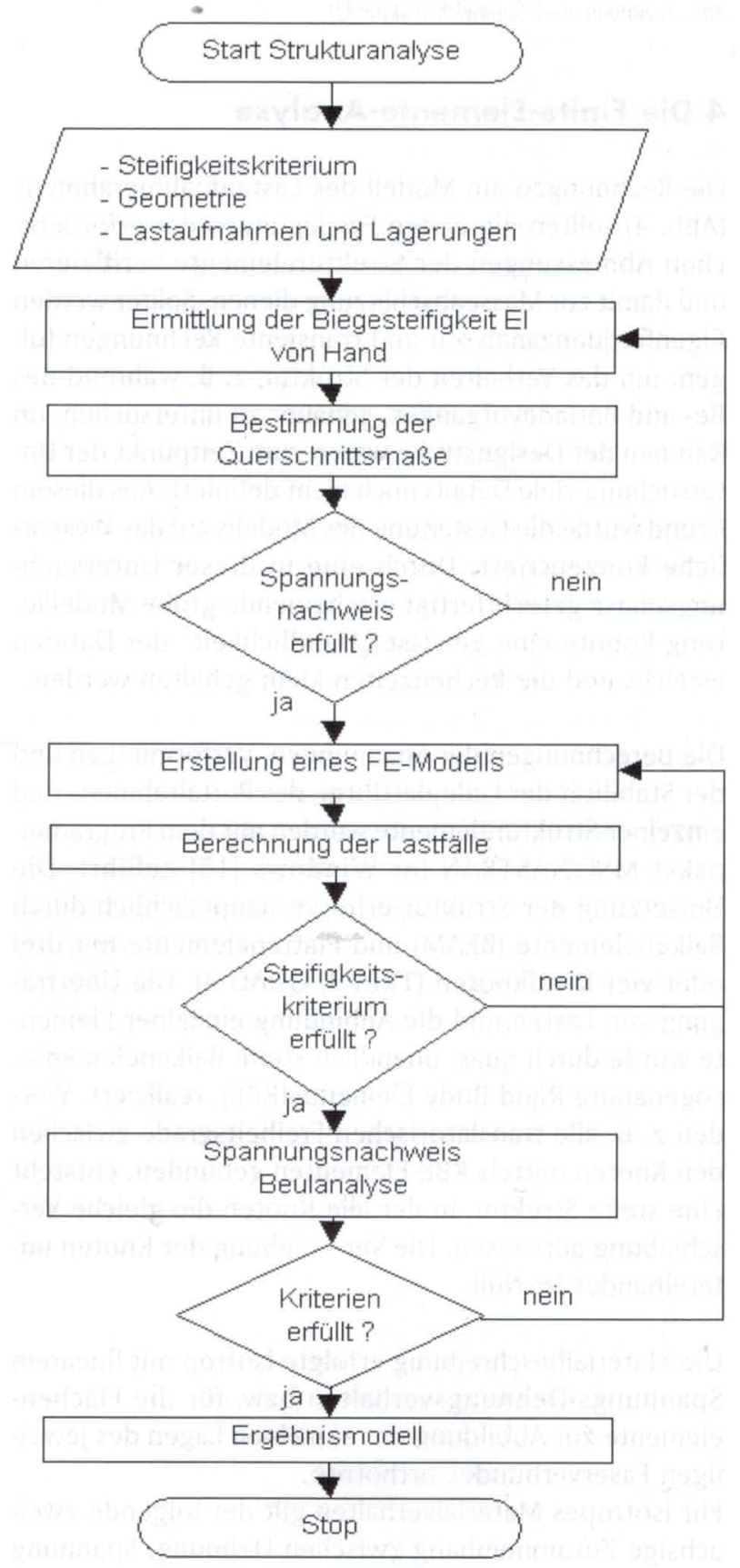

Abb. 6: Algorithmus der Modellerstellung 
Die auf diese Weise bestimmten Querschnitte waren als FE-Modelle zu approximieren und anschließend zu untersuchen. Dabei handelte es sich in der Metallbauweise um geschweißte I-Profile für die Längs- und Querträger der Ladeplattform und quadratische Kastenprofile für die Portalarme und Traversen aus luftfahrtzugelassenen Aluminiumblechen ALCu4Mg2.

Die Faserverbundbauweise wurde als Sandwichstruktur (Abb. 7) mit Aluminiumwaben (Honeycomb) als Kern und faserverstärkter Kunststoffhaut ausgefuihrt.

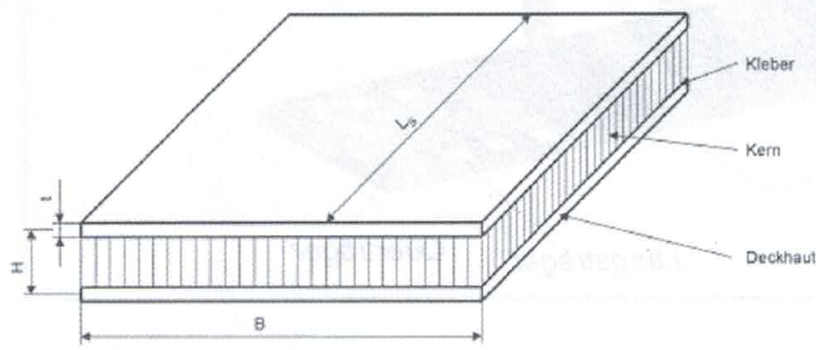

Abb. 7: Aufbau einer Sandwichstruktur [3]

\section{Die Finite-Elemente-Analyse}

Die Rechnungen am Modell des Lastaufnahmerahmens (Abb. 4) sollten die ersten Festlegungen der erforderlichen Abmessungen der Strukturelemente verifizieren und damit zur Masseabschätzung dienen. Später werden Eigenfrequenzanalysen und transiente Rechnungen folgen, um das Verhalten der Struktur, z. B. während des Be- und Entladevorganges, genauer zu untersuchen. Im Rahmen der Designstudie waren zum Zeitpunkt der Untersuchung viele Details noch nicht definiert. Aus diesem Grund wurde die Gestaltung des Modells auf das Wesentliche konzentriert. Durch eine in dieser Untersuchungsphase gerechtfertigt erscheinende grobe Modellierung konnte eine gewisse „Handlichkeit“ der Dateien erreicht und die Rechenzeiten klein gehalten werden.

Die Berechnungen der Spannungen, Verformungen und der Stabilität der Ladeplattform, des Portalrahmens und einzelner Strukturelemente wurden mit dem Programmpaket MSC/NASTRAN for Windows [15] geführt. Die Umsetzung der Struktur erfolgte hauptsächlich durch Balkenelemente (BEAM) und Plattenelemente mit drei oder vier Randknoten (TRIA 3, QUAD 4). Die Übertragung von Lasten und die Anbindung einzelner Elemente wurde durch quasi unendlich steife Balkenelemente, sogenannte Rigid Body Elements (RBE), realisiert. Werden z. B. alle translatorischen Freiheitsgrade zwischen den Knoten mittels RBE-Elementen gebunden, entsteht eine steife Struktur, in der alle Knoten die gleiche Verschiebung aufweisen. Die Verschiebung der Knoten untereinander ist Null.

Die Materialbeschreibung erfolgte isotrop mit linearem Spannungs-Dehnungsverhalten bzw. für die Flächenelemente zur Abbildung der einzelnen Lagen des jeweiligen Faserverbundes orthotrop.

Für isotropes Materialverhalten gilt der folgende zweiachsige Zusammenhang zwischen Dehnung, Spannung und Materialkennwerten.

$$
\left[\begin{array}{c}
\varepsilon_{1} \\
\varepsilon_{2} \\
\gamma_{12}
\end{array}\right]=\left[\begin{array}{ccc}
\frac{1}{E} & \frac{-v}{E} & 0 \\
\frac{-v}{E} & \frac{1}{E} & 0 \\
0 & 0 & \frac{1}{G}
\end{array}\right] \cdot\left[\begin{array}{c}
\sigma_{1} \\
\sigma_{2} \\
\tau_{12}
\end{array}\right]
$$

mit

$$
G=\frac{E}{2(1+v)}
$$

Für die Berechnung flächenartiger orthotroper Strukturen gilt:

$\left[\begin{array}{l}\varepsilon_{1} \\ \varepsilon_{2} \\ \gamma_{12}\end{array}\right]=\left[\begin{array}{ccc}\frac{1}{E_{1}} & \frac{-v_{12}}{E_{1}} & 0 \\ \frac{-v_{12}}{E_{1}} & \frac{1}{E_{2}} & 0 \\ 0 & 0 & \frac{1}{G_{12}}\end{array}\right] \cdot\left[\begin{array}{c}\sigma_{1} \\ \sigma_{2} \\ \tau_{12}\end{array}\right]$

mit

$v_{12} \cdot E_{2}=v_{21} \cdot E_{1}$

Im Folgenden werden exemplarisch Ergebnisse der FERechnung dargestellt.

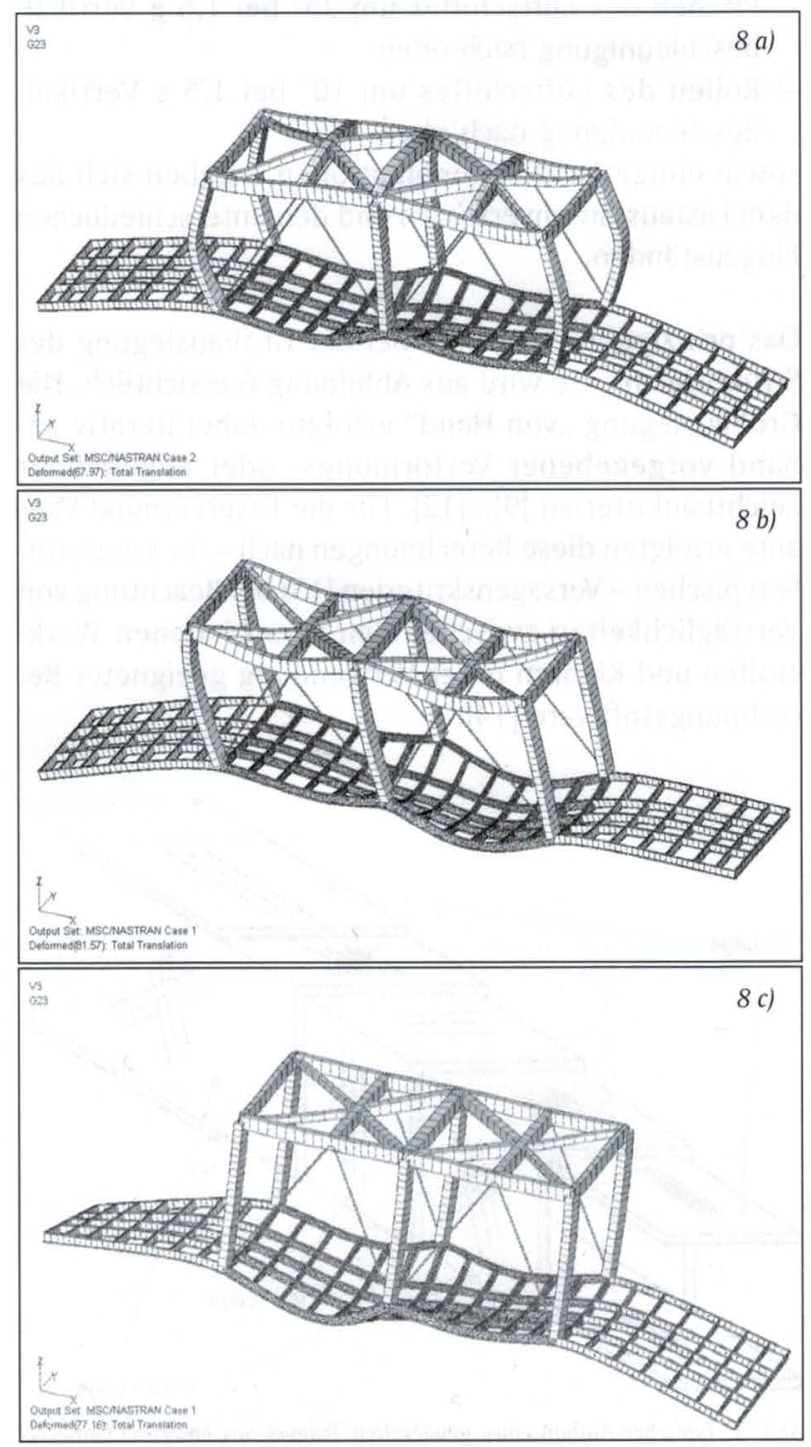

Abb. 8: Gesamtverformung des Lastaufnahmerahmens [4] 


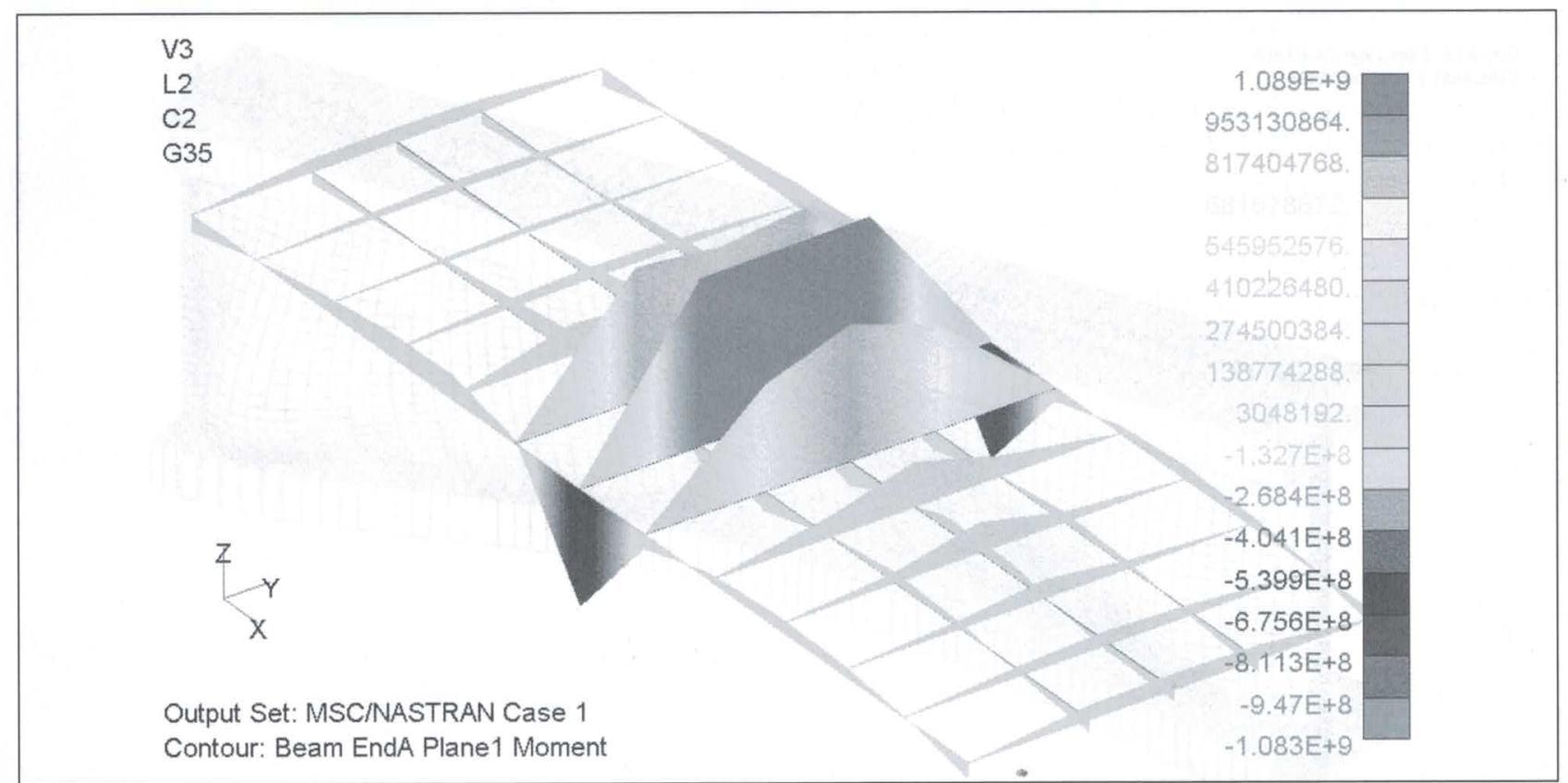

Abb. 9: Momentenverlauf der Ladeplattform [4]

Abbildung 8 zeigt die Gesamtverformung des Lastaufnahmerahmens in Metallbauweise bei verschiedenen Lastannahmen. Die Darstellung „a“ zeigt den verformten Lastaufnahmerahmen beim Herablassen des Ladesystems. Dabei werden Windböen sowie die Schubkraft der Vertikaltriebwerke, die zur Vermeidung des Abdriftens des Luftschiffs beim Lastausgleich benötigt wird, berücksichtigt. Die davon abweichenden Verformungen - vor allem des Portalrahmens - beim Pitchen und Rollen sind in den Bildern „b“ und „C" erkennbar. In den Abbildungen ist aus Gründen der besseren Erkennbarkeit auf die Darstellung der Deckplatten der Ladeplattform verzichtet worden. Die berechneten Verschiebungswerte für die einzelnen Lastzustände in den drei Ebenen erfüllen die definierten Steifigkeitskriterien.
Die mittels FE-Analyse berechneten Verformungen der Ladeplattform zeigen, wie erwartet, eine gute Übereinstimmung mit dem zur Grobabschätzung verwendeten einfachen Balkenmodell. Es hat sich gezeigt, dass die globalen Verformungen beider Berechnungsverfahren relativ gut übereinstimmen. Lokale Durchbiegungen, wie z. B. im Bereich der Lasteinleitungen, bzw. versteifende Wirkungen der momentenfesten Anbindung der Portalarme an die Ladeplattform können naturgemäß mit dem einfachen Balkenmodell nicht erfasst werden.

Die Rechnung hat auch gezeigt, dass der Einfluss der Steifigkeit der Ladeplattform auf die Verformung des Portals größer ist als umgekehrt. Die Reduzierung der Ladeplattformsteifigkeit als Ergebnis von Massereduzierung wird diese Verformung notwendigerweise ansteigen lassen (vgl. dazu auch Abb. 13).

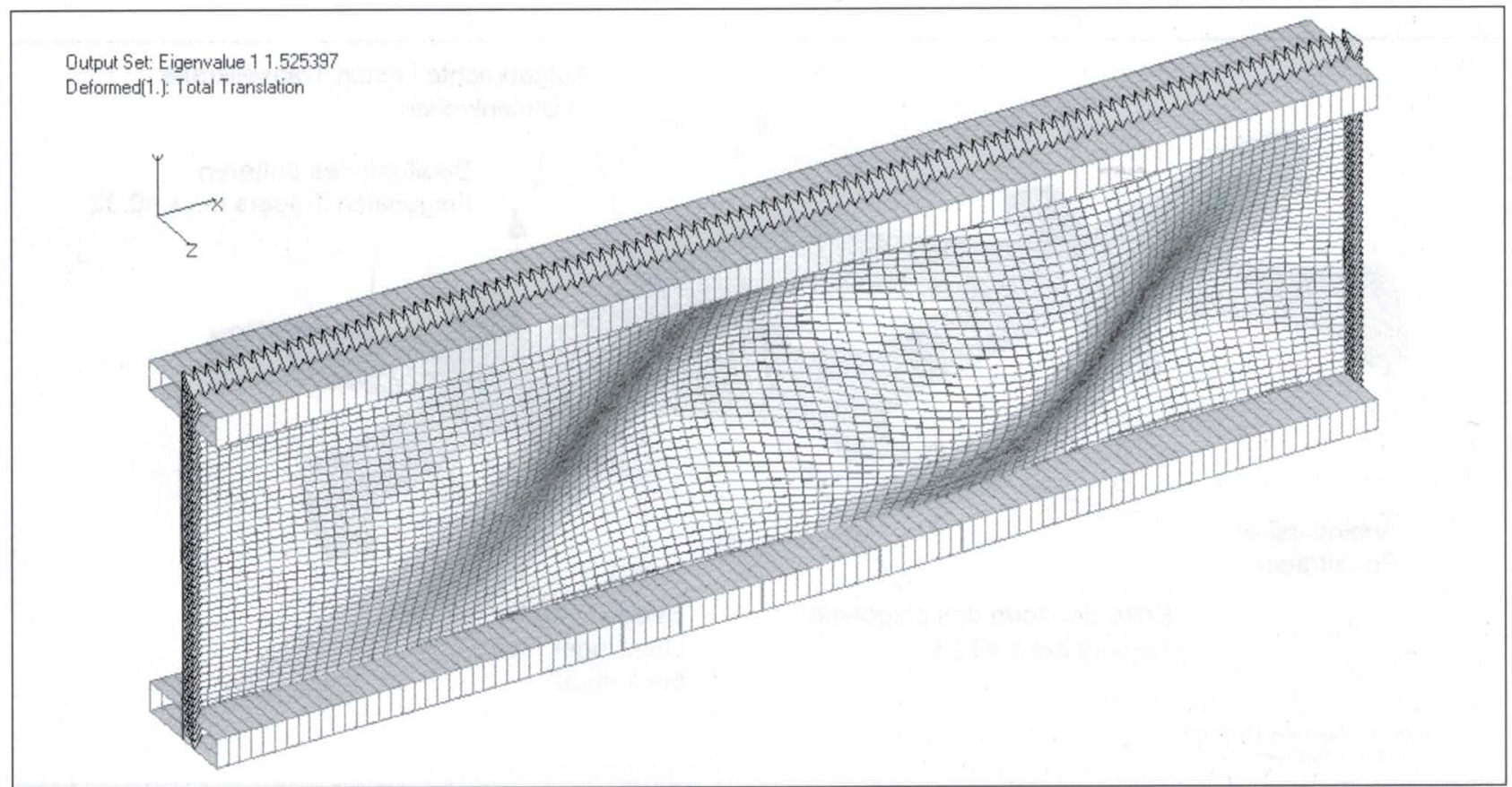

Abb. 10 a): Beulformen für Stabilitäts-Analysemodell [4] 


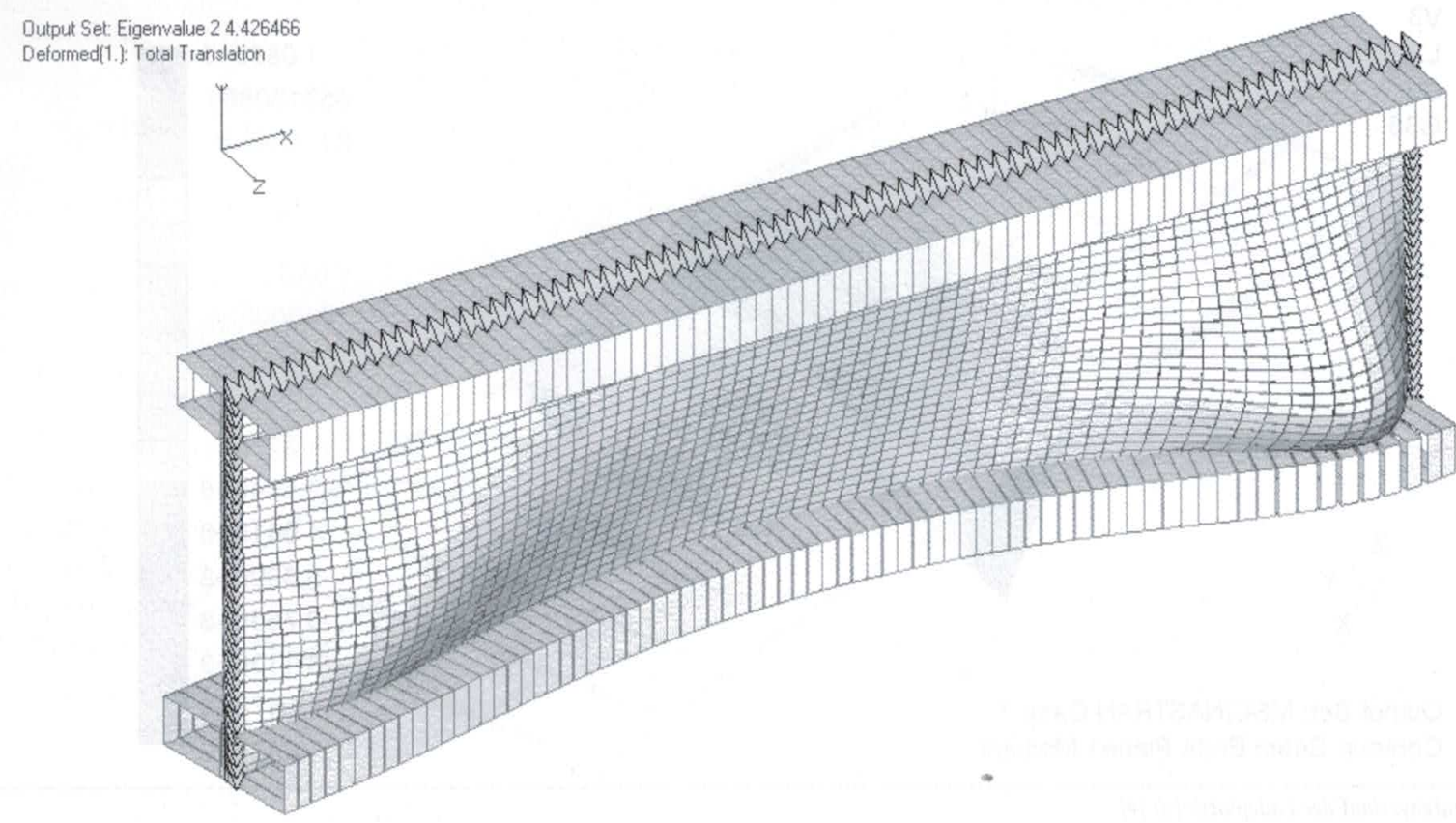

Abb. 10 b): Beulformen für Stabilitäts-Analysemodell [4]

Ebenso wurde deutlich, dass die beim Lastaustauschverfahren auftretenden Seilkräfte eine geringere Verformung verursachen als die vertikalen Beschleunigungen beim Pitchen und Rollen. Die Überlagerung des Pitchens und des Rollens führt dann zur maximalen Verformung des Portalträgers.

Hinsichtlich der Spannungen hat die FE-Rechnung gezeigt, dass die größten Materialbelastungen beim Rollen des Luftschiffes für die äußeren Längsträger im Bereich der mittleren Portalarme auftreten. Dies wird auch in Abbildung 9, in dem die Momentenverläufe der Ladeplattform dargestellt sind, erkennbar. Auch für die Gurte ergeben sich kritische Belastungen bei diesem Lastfall und z. T. auch beim Einholen des Ladesystems bei Berïcksichtigung von Windböen. Sämtli- che berechnete Spannungen liegen deutlich unterhalb kritischer Werte; die kleinste Sicherheit gegen das Fließen des verwendeten Aluminiumwerkstoffes z. B. wird $\mathrm{zu} \mathrm{v}_{\mathrm{F}}=2,3$ berechnet. Dies kann nicht verwundern, da ohnehin zumeist die Steifigkeits- und Stabilitätskriterien für die Bemessung der Bauteile maßgeblich sind.

Die Beulformen eines ausgewählten Querträgers der Ladeplattform in Metallbauweise bei verschiedenen Lastfällen, die anhand einfacher Submodelle mit konservativ angenommener Lagerung gewonnen wurden, werden exemplarisch in Abbildung 10 gezeigt. Die dabei ermittelten Eigenwerte entsprechen den Sicherheiten gegen Instabilität der Struktur bei den vorgegebenen Lasten.

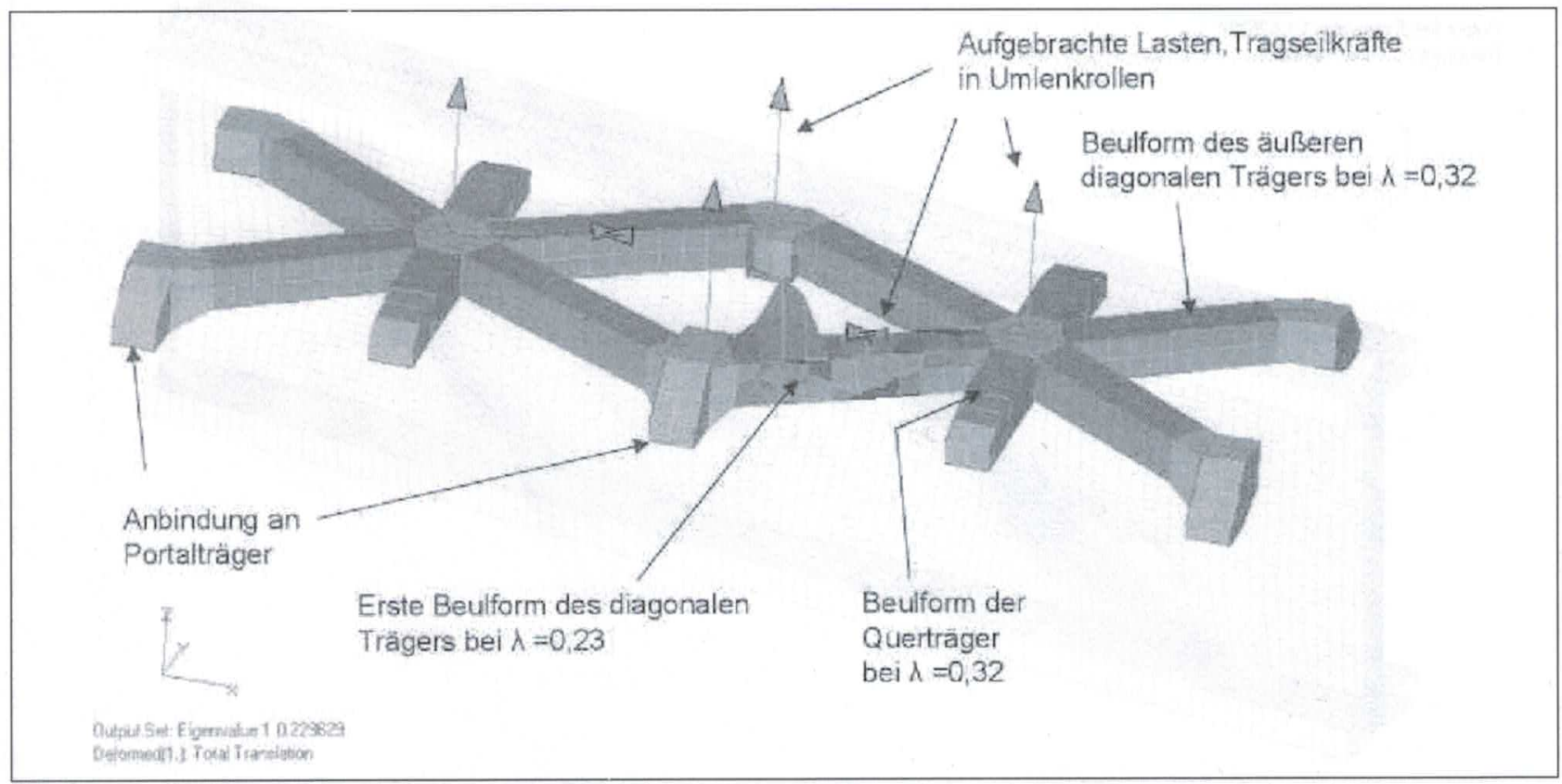


Nicht untersucht wurde die Tragfähigkeit der Struktur nach dem Ausbeulen. Eine solche Untersuchung müsste mittels einer nichtlinearen Analyse am Gesamtmodell, bei der Verformungen durch das Beulen zu berücksichtigen sind, geführt werden.

Bei der Stabilitätsuntersuchung von Elementen des Lastaufnahmerahmens aus faserverstärkten Kunststofflaminaten konnte das Versagen durch Ablöseerscheinungen der Deckhäute, hervorgerufen durch Beulen und Knittern mit den in der FE-Rechnung verwendeten QUAD-4Elementen, nicht untersucht werden. Diese Elemente bilden globale Beulformen der Sandwichstruktur und die Steifigkeit der Ladeplattform recht gut ab, indem ein verschmiertes E-Modul für die einzelnen Belastungsrichtungen anhand der einzelnen Lagen bestimmt wird. Der Fehler aufgrund der geringen Elementeanzahl kann für die maßgebliche erste Beulform analog der ersten Eigenform bei einer Modalanalyse, die auch schon bei grober Vernetzung hinreichend genaue Ergebnisse liefert, als gering angesehen werden.

Die Bètrachtung des Nachbeulverhaltens mit nichtlinearer Nachweisrechnung wurde mittels Submodelltechnik geführt, um den doch erheblichen Rechenaufwand zu reduzieren.

Die Beulformen eines Trägers aus der Sternsektion des Portals (erkennbar in Abb. 11) zeigt Abbildung 12. Die Berechnungen haben gezeigt, dass das kritische Kriterium die Beulsicherheit darstellt. So erhöhte sich die Gesamtmasse des Portals infolge notwendiger konstruktiver Änderungen aufgrund der Ergebnisse der Beulanalyse um ca. 24 \% gegenüber den Ausgangsannahmen.

Auf der anderen Seite sind in der Modellrechnung auch deutliche Reserven hinsichtlich der Ausnutzung der Steifigkeitskriterien einzelner Baugruppen deutlich geworden.
Dies war Veranlassung zu einer Modelloptimierung des Lastaufnahmerahmens in Faserverbundbauweise mit den folgenden Schwerpunkten:

- Veränderung der Lasteinleitung im Schwerlastbereich der Ladeplattform,

- Reduzierung der Lagendicken der Deckhäute der Ladeplattform,

- Reduzierung der Sandwichhöhe der Ladeplattform,

- Reduzierung der Wanddicke der Knotenbleche,

- Optimierung der Wanddicken der laminierten Träger.

Auf diese Weise ist eine Massereduzierung der Strukturmasse von $17 \%$ erreicht worden. Abbildung 13 zeigt Ergebnisse der Optimierungsstufen und lässt die gegenseitigen Abhängigkeiten der Strukturveränderungen deutlich werden. So wird die Massereduzierung der Ladeplattform um ca. $10.600 \mathrm{~kg}$ mit einer notwendigen Masseerhöhung des Portals um ca. 1.100 kg „erkauft“.

\section{Zusammenfassung und Ausblick}

Die in der Untersuchung gefuihrten umfangreichen iterativ-konventionellen und numerischen Berechnungen dienten der Bestimmung erster Strukturdaten zur Definition der Strukturmasse des Lastaufnahmerahmens in zwei verschiedenen Ausführungsformen.

Wie jede Untersuchung hat auch diese nicht nur (erste) Antworten gegeben, sondern eine Vielzahl von Fragen aufgeworfen und damit Aufgabenstellungen für weitere Analysen geliefert.

Auch sind die vorhandenen Modelle durch Konkretisierung der Anforderungen zu korrigieren, anzupassen und - wo nötig - zu verfeinern.

Die Massereduzierung besitzt bei der Konstruktion von Fluggeräten einen hohen Stellenwert. Da mit den zu be-

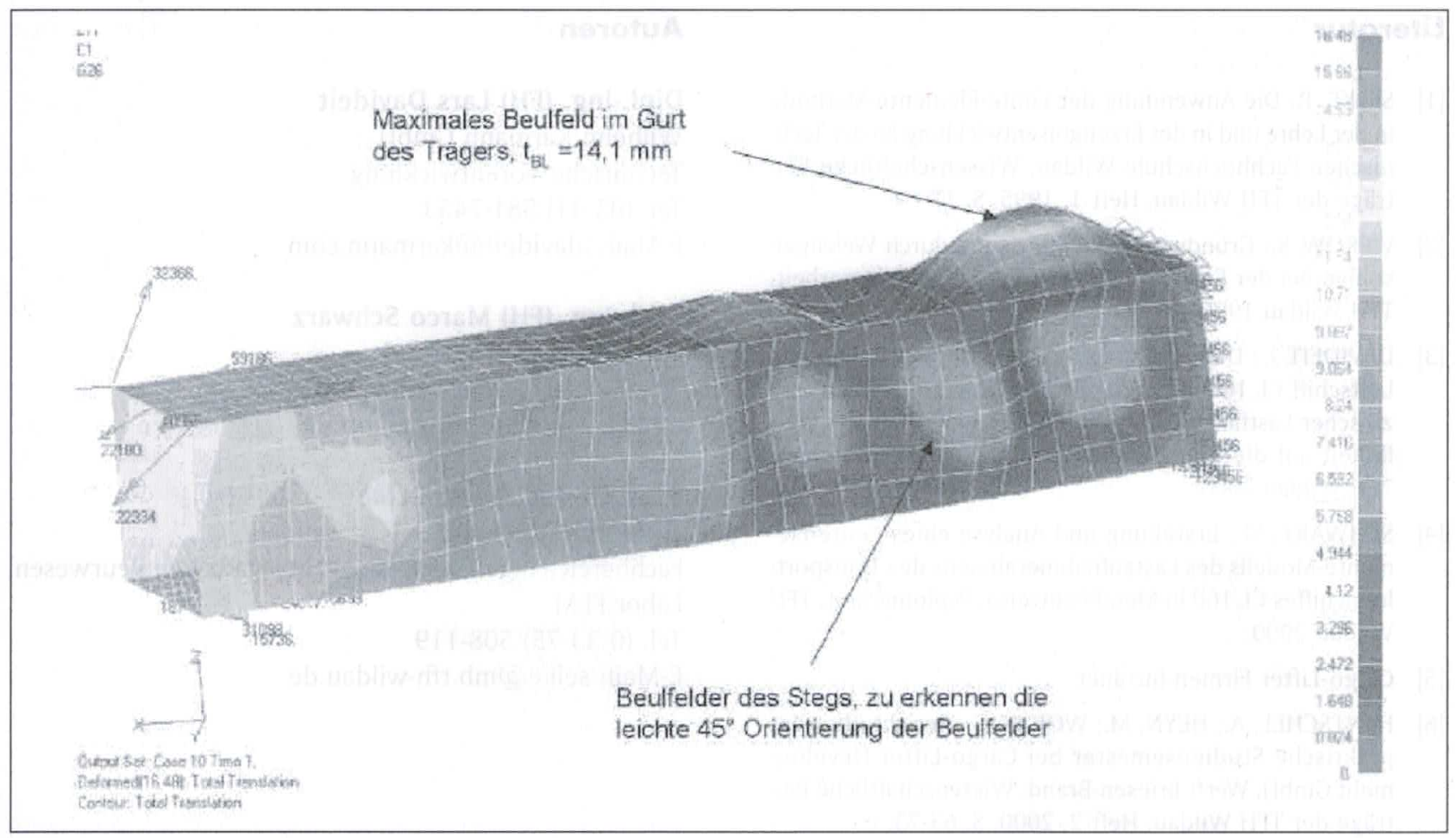

Abb. 12: Beulform der Sternträgersektion [3] 


\section{- Die Strukturmasse im Verlauf der Optimierung}

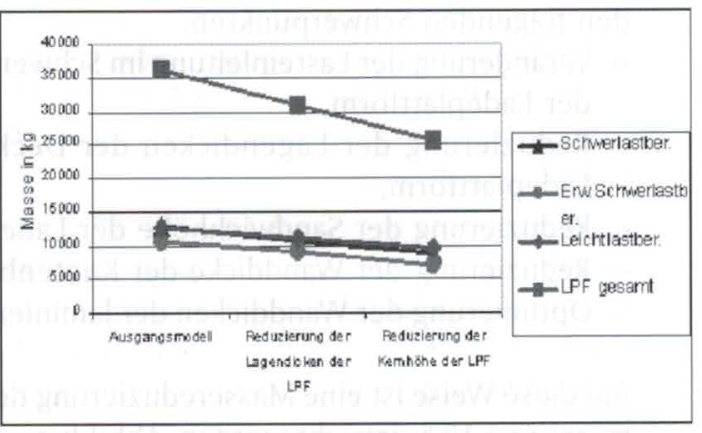

-Die Masse der Ladeplattform sinkt von $36173 \mathrm{~kg}$ auf $25578 \mathrm{~kg}$.

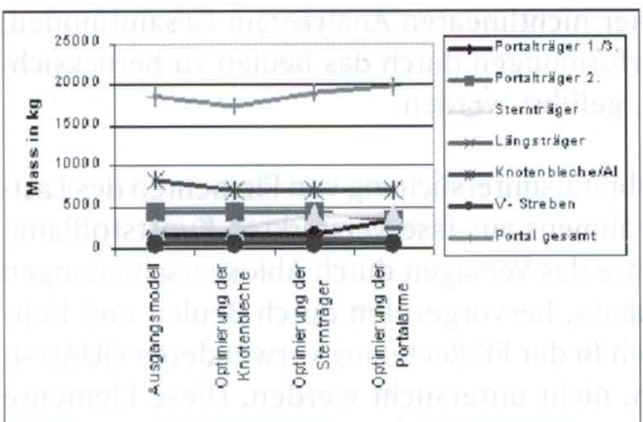

-Die Masse des Portals steigt von $18596 \mathrm{~kg}$ auf $19676 \mathrm{~kg}$.

\section{-Die Gesamtmasse des Laderahmens sinkt von 54,8 $\mathrm{t}$ auf 45,3t}

Abb. 13: Strukturmasse im Verlauf der Optimierung [3]

rüicksichtigenden Kriterien bei der Auslegung der Strukturelemente die obere Grenze der Strukturmasse ermittelt wurde, ist ein Potenzial an Gewichtseinsparung vorhanden. Mögliche Ansatzpunkte hierfür sind:

- Anpassung der Länge der Ladeplattform an die Hauptmaße des jeweiligen Ladegutes durch Segmentierung (modularer Aufbau).

- Optimierung der Kraftflüsse auf der Grundlage der gewonnenen Ergebnisse der Untersuchung durch Veränderung des Designs und der Anordnung der Seilrollen.

- Entwickeln eines Konzeptes zur Lastenverteilung der Transportgüter mit dem Ziel der Gewichtsreduzierung der Deckplatten der Ladeplattform.

- Querschnittsoptimierung auf der Grundlage verfeinerter Modellrechnungen.

- Untersuchung der Möglichkeit des Einsatzes einer Fachwerkkonstruktion.

\section{Literatur}

[1] SELKE, P.: Die Anwendung der Finite-Elemente-Methode in der Lehre und in der Erzeugnisentwicklung an der Technischen Fachhochschule Wildau, Wissenschaftliche Beiträge der TFH Wildau, Heft 1, 1995, S. 17-19.

[2] VIEROW, S.: Grundwasserbeeinflussung durch Weichgelsohlen bei der Errichtung von Baugruben, Diplomarbeit, TFH Wildau 1996.

[3] DAVIDEIT, L.: Dimensionierung des Laderahmens für das Luftschiff CL 160 in Faserverbund-Bauweise anhand spezifischer Lastfälle. Untersuchung des Einflusses der Steifigkeit auf die Masse des Laderahmens, Diplomarbeit, TFH Wildau 2000.

[4] SCHWARZ, M.: Erstellung und Analyse eines Finite-Elemente-Modells des Lastaufnahmerahmens des Transportluftschiffes CL 160 in Metallbauweise, Diplomarbeit, TFH Wildau 2000.

[5] Cargo-Lifter Firmen-Intranet

[6] HENTSCHEL, A.; HEYN, M.; WOJCIK, S.: Bericht über das praktische Studiensemester bei Cargo-Lifter Development GmbH, Werft Briesen-Brand, Wissenschaftliche Beiträge der TFH Wildau, Heft 2, 2000, S. 63-73.
[7] Produkțprospekt MAN TAKRAF Fördertechnik GmbH.

[8] VDI-Richtlinie: Entwicklung von Bauteilen aus FaserKunststoff-Verbund, Verein Deutscher Ingenieure 1989.

[9] HERTEL, H.: Leichtbau, Berlin, Heidelberg : Springer Verlag 1960.

[10] WIEDEMANN, J.: Leichtbau 1, Band 1 Elemente, Berlin, Heidelberg, New York: Springer Verlag 1996.

[11] WIEDEMANN, J.: Leichtbau 1, Band 2 Konstruktion, Berlin, Heidelberg, New York: Springer Verlag 1996.

[12] KLEIN, B.: Leichtbau-Konstruktion, Braunschweig: Vieweg Verlag 1998.

[13] HFF: Gestaltungshinweise zu Sandwich-Laminaten, MK Helicopter GmbH 1999.

[14] Math Soft/Mathcad 8, Benutzerhandbuch, Bonn: MITP Verlag GmbH 1998.

[15] Mac Neal - Schwendler Corporation.

\section{Autoren}

\section{Dipl.-Ing. (FH) Lars Davideit}

Wilhelm Karmann GmbH

Technische Vorentwicklung

Tel. (05 41) 581-7453

E-Mail: ldavideit@karmann.com

Dipl.-Ing. (FH) Marco Schwarz

Rolls-Royce Deutschland

Whole Engine Mechanics

E-Mail: Marco.Schwarz@brr.de

\section{Prof. Dr.-Ing. Peter Selke}

Technische Fachhochschule Wildau

Fachbereich Ingenieurwesen/Wirtschaftsingenieurwesen, Labor FEM

Tel. (0 33 75) 508-119

E-Mail: selke@mb.tfh-wildau.de 